\title{
Casting of concrete and reinforced concrete pipes by vibro-vacuum technique
}

\author{
Basheer Younis*, Ibragim Kazimahomedov, Saad Salem, Tatiana Kostuk and Elena \\ Dedenyova \\ Kharkiv National University of Civil Engineering and Architecture, 61002 Sumska st, 40, Kharkiv, \\ Ukraine
}

\begin{abstract}
This article deals with the casting of concrete and reinforced concrete pipes by vibro-vacuum technique. The existing techniques are investigated, the shortcomings are revealed and the solutions to major issues with the improved techniques were offered. Improvement of a technological process of concrete and reinforced concrete pipes production is an important and current task. Development of such technologies should ensure the required supporting capacity reducing the cost of pipeline construction thereof. In this article the existing ways of molding concrete and reinforced concrete pipes have been evaluated. Their engineering deficiencies in terms of labour-intensity and obtaining the products with increased physical and mechanical characteristics have been identified. The offered solution addressing the problems has been identified, namely, the casting of concrete pipes by vibro-vacuum technique. Pilot studies have confirmed that this method makes it possible to achieve the sufficiently high physico-mechanical characteristics of the products. Tensile strength of vibro-vacuum pipes is $40 \%$ higher than that of pipes obtained by layerby-layer axial compaction.
\end{abstract}

\section{Introduction}

The modern requirements to the quality of construction products are not inferior to the demands for the newest architectural elements $[1,2]$. In addition to the high requirements for physico-mechanical characteristics and durability of concrete and reinforced concrete pipes, the requirements for the quality and casting techniques are set. The casting process consists of assembling molds, fixture installation, laying and compaction of concrete mix.

The most useful casting technique is determined in accordance with design features of the concrete products, technological production flow diagram, degree and type of reinforcement, mass and dimensions of the products, quality requirements for casting and compacting of concrete mix, quality requirements for the surface of the product, availability and volume of production [1-6].

Currently, in the construction market there is a steady trend towards automated production. For laying and compacting of concrete mix the following techniques are

*Corresponding author: docbasheer01@gmail.com 
applied: vibrating, vibro-stamping, vibro-rolling, vibro-vacuum, centrifugation, etc., as well as the combined ones, such as: concrete vibrating and pressing, centrifuging, rolling, etc. [7-10].

The purpose of the article is to investigate the current requirements for construction products, the casting techiques and their relevance so far, to identify the negative aspects of existing techniques and to suggest the ways to solve them in terms of operation and product quality.

\section{Analysis of publications on the research topic}

The article is based on studies in the field of building materials and casting techiques for pipelines by Yu.M.Bazhenov [6], A.G. Komar B.G., Skramtaev [7], A.E. Desov et al. The analysis of these works allows considering the casting techiques for the pipelines, in particular, vibro-vacuum, but they are not enough for an integrated approach to pipeline systems upgrading. Analysis of these works allows considering the casting techiques for the pipelines, to determine their efficiency, accompanied by an integrated approach to improving physical and mechanical characteristics of concrete and reinforced concrete pipes . The article is based on studies in the field of production of concrete pipes by I.N. Akhverdova V.I. Babushkin, A.G. Vandolovsky, V.N. Ladyzhensky, O.P. MchedlovPetrosyan, A.E. Shmurnov, B.B. Khasanov, A.N. Popov, P.A. Makarov et al. The two methods of compacting a concrete mix with a core rotated inside the mold have proliferated in the production of concrete pipes: rotational layer-by-layer pressing and radial pressing.

The rotational pressing is made by pressing a curved blade sliding over the compacted concrete, while it falls under sliding friction and wears out pretty quickly.

For the layer-by-layer radial pressing a sealing core with a roller system on the pressing core instead of the blade increasing the service life has been developed. An important condition is the rotation of the rollers during compaction without sliding which causes certain technological difficulties.

The proposed vibro-vacuum sealing technique in comparison with previous methods makes it possible to obtain the pipes and shaped elements of increased strength characteristics, resulting in the increased the service life of pipelines.

\section{The basic content of the article}

There is a casting technique for dry-concrete mixes in molds with one open surface by feeding a concrete mix along a number of identical, parallel, freely rotating blades on the axes contacting the exposed surface of the molded product and moving relative to the open surface of the mold in the transverse and longitudinal directions. Moreover, the concrete mix is fed under the blades by a layer exceeding the thickness of the molded article under the blade, and the speed of the blades moving along their axes is selected, depending on the speed of the layer of the concrete mix being squeezed out from under the front ends of the blades along the axis of their movement. However, such techniques do not guarantee the high quality of the molded articles. In the same pipe, areas of the highest quality of compaction may be along with insufficiently compacted ones. The reason for this is insufficient control and the impossibility of maintaining a constant layer of loose concrete mix over the blade head.

The closest to the claimed technical solution is the precast-concrete pipes casting technique where the concrete mix is moistened in the space between the mold and the core and compact the mix due to excessive pressure in the core with simultaneous vibration. The excessive pressure is created by water injection between the rubber sleeve and the metal 
core from the inside. Preserving the excess pressure of the rubber stocking on the concrete mixture, the mold is directed to heat treatment. The shortcomings of the above method include the injection of concrete mix into the interstice space from below due to excess pressure resulting in additional labor costs and other problems.

The task is to increase the productivity and quality of molded concrete and reinforced concrete pipes. To solve the existing problem, it is proposed to use the vibro-vacuum technique in casting of concrete and reinforced concrete pipes in the building industry.

Vibro-vacuuming is a casting technique where vibration of a concrete mix, carried out by one of the known techniques, is combined with the removal of air and excess water from it. Vibro-vacuuming is a complex way of casting products, including vacuum dewatering and repeated short-term vibration. Casting of products is carried out in vertical and horizontal facilities, providing compaction of the concrete mix, immediate stripping and unattended (on the sucker) removal of freshly molded products.

In connection with the fact that apart from the pipes there is a need for branch pipe parts (Y-branch fittings) in pipelines a technological solution for casting of complex shaped products from high-flow concrete mixes was developed.

Close to the developed technical solution is the precast-concrete pipes casting technique where the concrete mix is moistened in the space between the mold and the core and compact the mix due to excessive pressure in the core with simultaneous vibration. The excessive pressure is created by water injection between the rubber sleeve and the metal core. In the production of pipes this way the upper fed high-flow concrete mixes the mold are used.

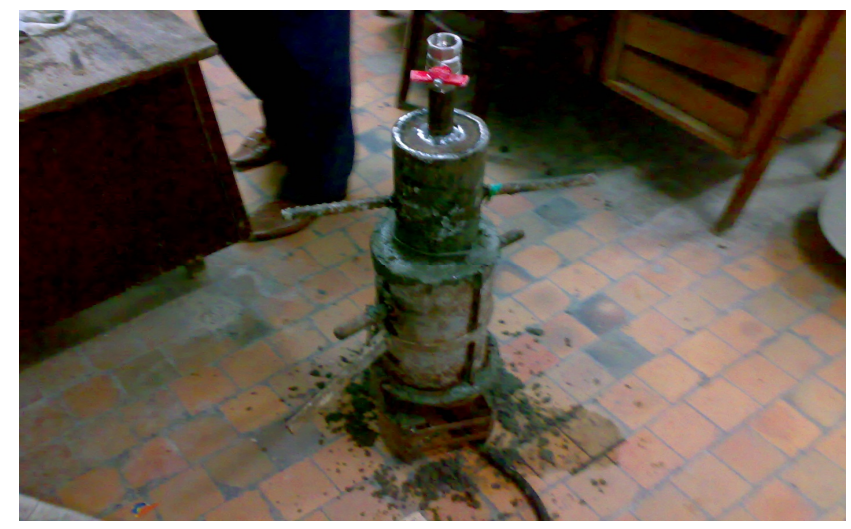

Fig. 1. Laboratory facility for the production of concrete and reinforced concrete pipes by vibrovacuum technique.

It has been established by experimental means that the highest compaction quality of the pipes is achieved using the vibro-vacuum casting technique where up to $30 \%$ of excessive water is removed from freshly placed concrete mix, as a result of this treatment, the concrete mix is compacted, which in turn improves its properties, namely, tensile strength. The figures obtained are presented in Table 1.

Table 1. Tensile strength of concrete pipes

\begin{tabular}{|c|c|c|}
\hline \multicolumn{2}{|c|}{ Tensile strength of concrete pipes $\left(\mathrm{R}_{\mathrm{bt}}\right) \mathrm{MPa}$} & \multirow[b]{2}{*}{$\mathrm{W} / \mathrm{C}$} \\
\hline $\begin{array}{c}\text { Samples of pipes obtained by layer-by- } \\
\text { layer axial compaction }\end{array}$ & $\begin{array}{l}\text { Samples of pipes obtained by vibro- } \\
\text { vacuum technique }\end{array}$ & \\
\hline 4.20 & 6.32 & 0.5 \\
\hline 3.93 & 6.80 & 0.55 \\
\hline 3.12 & 7.41 & 0.65 \\
\hline
\end{tabular}


The tensile strength of vibro-vacuum pipes is $40 \%$ higher than that of pipes obtained by layer-by-layer axial compaction. Simultaneously with vacuum treatment the concrete mix is compacted by vibration to increase its strength.

\section{Conclusion}

The study highlighted the main problems of quality and operation of concrete and reinforced concrete pipelines with existing casting techniques. The offered casting technique improves the characteristics of the products, in particular tensile strength. This technique is intended for casting of concrete and reinforced concrete pipes, rings, pillars of lighting supports and overhead contact system of concrete mixes.

\section{References}

1. R.F. Runova, V.I. Gotz, M.A. Sanytsky, Design materials of the new generation and technology in the future in the future (2008)

2. Z. Jamrozy, Concrete and its technologies (Scientific publishing house Pwn., Warsaw, 2000)

3. N. Zaichenko, High-strength fine-grained concrete with a complex microstructure of the modified (2009)

4. Yu. Bazhenov, Vysokoprochnyy melkozernistyy beton dlya armotsementnykh konstruktsiy (Moscow, 1963)

5. Akhverdov, Osnovy fiziki betona (Moscow, 1981)

6. Yu.M. Bazhenov, Technology of concrete and reinforced concrete products (Moscow, 1984)

7. B.G. Skramtaev, Building industry, 3, 64-72 (1938)

8. A.G. Vandolovsky, E.B. Dedeneva, Scientific Herald of Construction, 47, 192-195 (Kharkiv, 2008)

9. E. Dedeneva, I. Kazimagomedov, Saad Salem, T. Kostyuk, Basheer N. Younis, M. Yakimenko, MATEC Web Conf., 116 (2017)

10. B.N. Younis, E.B. Dedeneva, T.O. Kostiuk, , Proceedings of III International scientific conference, 28-31 (Morrisville ,2017) 